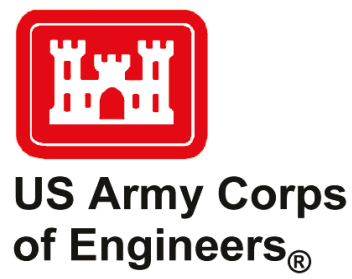

\title{
Formulation, Implementation and Testing of the Diffusive Wave (DW) Module of Adaptive Hydraulics (AdH)
}

\author{
by Gaurav Savant, Corey J. Trahan, and Tate O. McAlpin
}

PURPOSE: This Coastal and Hydraulics Engineering Technical Note (CHETN) describes the implementation and application of the Diffusive Wave (DW) module of the AdH numerical code, referred to as AdH-DW, for the rapid simulation of dam-break- and riverflow-caused flooding.

MOTIVATION AND BACKGROUND: Numerical simulation of inundation has been an integral part of flooding analysis since the advent of modern computing. These simulations have historically been performed using the Shallow Water Equations (SWE) in two dimensions (2D) due to the accuracy of results obtained. However, the solution of 2D-SWE can impose run-time constraints because of the computational intensity required (Prestininzi 2008); therefore, in general, 2D-SWE are of reduced use for problems where inundation results are required in a time-sensitive manner. In such instances, the computational efficiency and stability afforded by DW equations are of benefit.

DW equations (sometimes referred to as the non-inertia wave equations) utilize the SWE under the assumption that the inertial, pressure, and viscous terms are much smaller than the gravity and friction terms; such flows occur during fluvial flooding associated with dam and levee breach. Under these assumptions, the shallow water mass conservation equation simplifies to

$$
\frac{\partial h}{\partial t}+\frac{\partial h k \frac{\partial \varsigma}{\partial x}}{\partial x}+\frac{\partial h k \frac{\partial \varsigma}{\partial y}}{\partial y}=0
$$

where:

$$
\begin{aligned}
h= & \text { depth of fluid } \\
\varsigma & =h+Z b=\mathrm{SWE} \\
Z b= & \text { bed elevation } \\
k= & \frac{c^{2} h^{4 / 3}}{n^{2}\|V\|} \text { or } \frac{2 g h}{C_{d}\|V\|} \\
n= & \text { user-specified Manning's coefficient } \\
c= & \text { user-specified conversion factor }(1 \text { for S.I.; } 1.487 \text { for English units; or arbitrary } \\
& \text { conversion) } \\
C_{d}= & \text { drag coefficient } \\
\|V\| & =\text { velocity magnitude. }
\end{aligned}
$$


The momentum equations simplify to

$$
u=k \frac{\partial \varsigma}{\partial x}
$$

and

$$
v=k \frac{\partial \varsigma}{\partial y}
$$

where $u$ and $v$ are the $x$ - and $y$-direction velocity components, respectively.

This equation set has only one independent variable, $h$, and is hence a faster and easier solve than the full set of SWE. It has been shown that the DW equations yield reasonably good approximations for downstream backwater effects as well as depth of flooding and is thus useful when the speed of analysis is critical. Another benefit of using DW equations is that the solution is shock free and hence does not require the intensive numerical treatment that 2D-SWE require for stability purposes.

LIMITATIONS OF DW EQUATIONS: The diffusive wave equations are obtained by assuming that the inertial and pressure forces are insignificant when compared to the friction and slope terms.

These assumptions imply that in areas of extreme slope change and areas where pressure forces might be significant, such as at the site of a breach, the velocity results provided by DW equations will be invalid; however, the overall inundation extents might be correct. In view of the limitations listed, the authors urge extreme care in deciding whether $D W$ equations are appropriate for the problem under consideration.

FINITE ELEMENT DISCRITIZATION: AdH-DW discretizes the equations of mass conservation using a finite element (FE) approach. The exact FE discretization in AdH-DW uses a Galerkin Least Squares (GLS) approach with linear weight and basis functions on linear triangles. This section describes how this discretization is achieved and implemented in AdH-DW.

Basics. The FE discretization is performed over a triangular element mapped to a computational parent $\{(0,0) ;(0,1) ;(1,0)\}$ as represented in Figure 1.

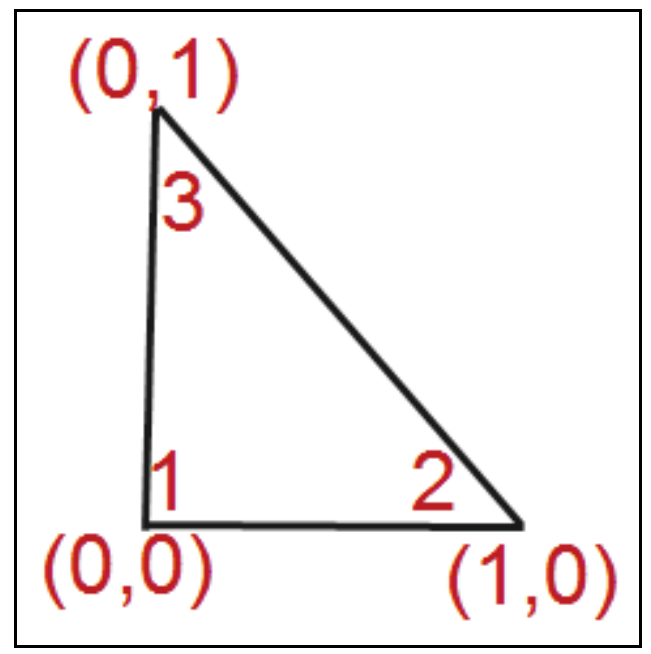

Figure 1. Computational element. 
The linear basis functions $\left(\phi_{l}\right)$ are then defined as

$$
\begin{aligned}
& \phi_{1}=1-\xi-\eta \\
& \phi_{2}=\xi \\
& \phi_{3}=\eta
\end{aligned}
$$

where $\xi$ and $\eta$ are locally defined variables whose values vary linearly from 0 to 1 (Figure 2) (i.e., (1) at node 1 the value of $\xi$ and $\eta$ is 0 ; and therefore $\phi_{1}$ is equal to $1, \phi_{2}$ and $\phi_{3}$ are equal to 0 ; (2) at node 2 the value of $\xi$ is 1 and $\eta$ is 0 ; and therefore $\phi_{2}$ is equal to $1, \phi_{1}$ and $\phi_{3}$ are equal to 0 ; (3) at node 3 the value of $\xi$ is 0 and $\eta$ is 1 ; and therefore $\phi_{3}$ is equal to $1, \phi_{1}$ and $\phi_{2}$ are equal to 0 ).

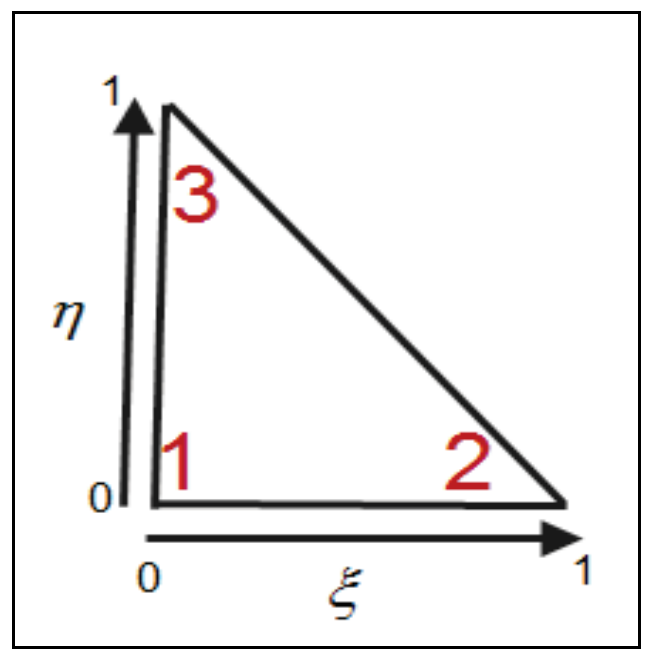

Figure 2. Variation in $\zeta$ and $\eta$.

In traditional finite element fashion, the linear basis functions are used to expand any solution variables so that, for three node triangles, the continuous field discretizes to

$$
\begin{gathered}
x=\sum_{1}^{3} x_{i} \phi_{i}(\xi, \eta)=x_{1} \phi_{1}(\xi, \eta)+x_{2} \phi_{2}(\xi, \eta)+x_{3} \phi_{3}(\xi, \eta) \\
y=\sum_{1}^{3} y_{i} \phi_{i}(\xi, \eta)=y_{1} \phi_{1}(\xi, \eta)+y_{2} \phi_{2}(\xi, \eta)+y_{3} \phi_{3}(\xi, \eta) \\
h(t)=\sum_{1}^{3} h_{i}(t) \phi_{i}(\xi, \eta)=h_{1}(t) \phi_{1}(\xi, \eta)+h_{2}(t) \phi_{2}(\xi, \eta)+h_{3}(t) \phi_{3}(\xi, \eta)
\end{gathered}
$$

where all variables are as previously defined.

Equations 5 through 7 above expand the variables in the computational or parent space. The expansion in the physical space is written as 


$$
\begin{gathered}
x=\sum_{1}^{3} x_{i} \hat{\phi}_{i}(x, y)=x_{1} \hat{\phi}_{1}(x, y)+x_{2} \hat{\phi}_{2}(x, y)+x_{3} \hat{\phi}_{3}(x, y) \\
y=\sum_{1}^{3} y_{i} \hat{\phi}_{i}(x, y)=y_{1} \hat{\phi}_{1}(x, y)+y_{2} \hat{\phi}_{2}(x, y)+y_{3} \hat{\phi}_{3}(x, y) \\
h(t)=\sum_{1}^{3} h_{i}(t) \hat{\phi}_{i}(x, y)=h_{1}(t) \hat{\phi}_{1}(x, y)+h_{2}(t) \hat{\phi}_{2}(x, y)+h_{3}(t) \hat{\phi}_{3}(x, y)
\end{gathered}
$$

where $\hat{\phi}$ is the basis function in the physical space and $h(t)$ is time varying depth. Hereafter, $h$ will be used to refer to the time varying quantity.

The transformation from the computational element to the physical element utilizes the Jacobian defined as

$$
J(\xi, \eta)=\left(\begin{array}{ll}
\frac{\partial x}{\partial \xi} & \frac{\partial x}{\partial \eta} \\
\frac{\partial y}{\partial \xi} & \frac{\partial y}{\partial \eta}
\end{array}\right)
$$

The relationship between the physical element area, denoted as " $\Lambda$ ", and J is

$$
\Lambda=\frac{1}{2}|J(\xi, \eta)|
$$

Since in typical FE fashion all integrations are performed on the computational element, the following transformations are written, as example, to map back and forth between the two:

$$
M=\int_{\Omega} \hat{\phi}_{i}(x, y) \hat{\phi}_{j}(x, y) d \Omega=\int_{\xi=0}^{1} \int_{\eta=0}^{1-\xi} \phi_{i}(\xi, \eta) \phi_{j}(\xi, \eta)(2 \Lambda) d \xi d \eta
$$

and

$$
K=\int_{\Omega} \frac{\partial \hat{\phi}_{i}}{\partial x} \frac{\partial \hat{\phi}_{j}}{\partial y} d \Omega=\frac{1}{2 \Lambda} \int_{\xi=0}^{1} \int_{\eta=0}^{1-\xi}\left(\frac{\partial \phi_{i}}{\partial x}\right)\left(\frac{\partial \phi_{j}}{\partial y}\right) d \xi d \eta
$$

Weak Form. To be able to utilize Equation 1, it is recast in the weak form as

$$
\int_{\Omega}\left(w \frac{\partial h}{\partial t}-\frac{\partial w}{\partial x} h k \frac{\partial \varsigma}{\partial x}-\frac{\partial w}{\partial y} h k \frac{\partial \varsigma}{\partial y}\right) d \Omega+\int_{\Gamma} w\left(h k \frac{\partial \varsigma}{\partial x} n_{x}+h k \frac{\partial \varsigma}{\partial y} n_{y}\right) d \Gamma=0
$$


where:

$$
\begin{aligned}
\Omega & =\text { global domain } \\
\Gamma & =\text { the global boundary } \\
n_{x} \text { and } n_{y} & =\text { outward normal in the } x \text { - and } y \text {-directions, respectively. }
\end{aligned}
$$

The weight function, $w$, is an arbitrary function that is smooth and has compact support. Since $w$ is arbitrary, any solution to Equation 1 is a solution to the weak form as well. Note that $w$ is being used to enforce the essential boundary conditions as well.

Until this point, the solutions are exact while the sections below expand the weak form into the finite element statement such that numerical solutions can be obtained.

The only dependent variable, $h$, is interpolated on the individual elements using linear Lagrange polynomials:

$$
h_{h}(x, y, t)=\sum_{i} \phi_{i}(x, y) h_{i}(t)
$$

where the subscript $i$ indicates the nodal value and the subscript $h$ indicates the approximate value. Henceforth the subscript $h$ is dropped, but it should be understood that all values are approximate.

Pure Galerkin FE methods have been shown to have spurious oscillations in convectiondominated systems when certain combinations of elements and weight functions are used. The combination of linear triangles and linear basis functions used in AdH is one such case. Various strategies such as the Streamline Upwind Petrov-Galerkin, Galerkin Least Squares (GLS), etc., exist that avoid the generation of spurious oscillations by enriching the Galerkin weight function, usually by adding a discontinuous weight function to the continuous Galerkin weight function. AdH-DW uses the GLS strategy to suppress these oscillations. The section below provides a description of the continuous and discontinuous parts of the weight function.

The finite element form is obtained by multiplying Equation 1 by a GLS weight function, $\psi=\phi+\varphi$, trial + GLS function and integrating by parts over the domain to obtain the following:

$$
\bar{R}=\sum_{e}\left[\int_{\Omega_{e}}\left(\phi_{i} \frac{\partial h}{\partial t}-\frac{\partial \phi_{i}}{\partial x} h k \frac{\partial \varsigma}{\partial x}-\frac{\partial \phi_{i}}{\partial y} h k \frac{\partial \varsigma}{\partial y}\right) d \Omega_{e}+\int_{\Gamma \cap \Gamma_{e}} \phi_{i}\left(h k \frac{\partial \varsigma}{\partial x} n_{x}+h k \frac{\partial \varsigma}{\partial y} n_{y}\right) d \Gamma_{e}+\bar{G}\right]=0
$$

where:

$$
\begin{aligned}
& \Omega_{\mathrm{e}}=\text { elemental } 2 \mathrm{D} \text { domain } \\
& \Gamma_{\mathrm{e}}=\text { elemental boundary } \\
& \Gamma=\text { the global boundary } \\
& \bar{G}=\text { the GLS contribution. }
\end{aligned}
$$




$$
\bar{G}=\tau \varphi\left(\frac{\partial h_{e}}{\partial t}+u_{e} \frac{\partial h_{i}}{\partial x}+v_{e} \frac{\partial h_{i}}{\partial y}\right)
$$

where the subscript $e$ indicates an elemental average. The GLS weight function takes the following form:

$$
\varphi=\left(u_{e} \frac{\partial \phi_{i}}{\partial x}+v_{e} \frac{\partial \phi_{i}}{\partial y}\right)
$$

$\tau$ is a weighing function, and the form it takes in AdH-DW is

$$
\tau=\frac{l_{e}}{\sqrt{u_{e}^{2}+v_{e}^{2}}}
$$

where $l_{e}$ is the spectral radius, and in AdH-DW, it is equivalent to the square root of the elemental area.

Notice that the discontinuous portion, $\varphi$, is composed of gradients of the continuous trial function and is only integrated against the discrete equation over the interior of the element. This means that essential boundary conditions are addressed by the continuous portion, $\phi$, of the test/weight function.

The sections below detail the integration of the continuous portion of the test/weight function against individual terms.

Continuity Equation. This section describes the FE discretization of the continuity equation (Equation 17).

\section{Temporal Term}

The temporal term is expressed using a finite difference, Crank-Nicholson, expression as

$$
\left(\frac{\partial h}{\partial t}\right)_{i}^{t+1}=\alpha\left[\frac{\left(\frac{3}{2} h_{i}^{t+1}-\frac{1}{2} h_{i}^{t}\right)-\left(\frac{3}{2} h_{i}^{t}-\frac{1}{2} h_{i}^{t-1}\right)}{\Delta t}\right]+(1-\alpha)\left[\frac{\left(h_{i}^{t+1}-h_{i}^{t}\right)}{\Delta t}\right]
$$

where:

$$
\begin{aligned}
& \alpha=\text { factor that determines order of time-stepping, } 0 \leq \alpha \leq 1 \\
& \Delta t=\text { time-step size. }
\end{aligned}
$$

Each term in Equation 21 is integrated consistently to obtain 


$$
\frac{1}{\Delta t} \int_{\Omega} \phi_{i} h^{n} d \Omega=\frac{1}{\Delta t} \int_{\xi=0}^{1} \int_{\eta=0}^{1-\xi} \phi_{i}\left(\phi_{1} h_{1}^{n}+\phi_{2} h_{2}^{n}+\phi_{3} h_{3}^{n}\right)(2 \Lambda) d \eta d \xi
$$

where:

$$
n=\text { the time under consideration }(\mathrm{t}-1, \mathrm{t} \text { or } \mathrm{t}+1) \text {. }
$$

\section{Convective Storage/Change Term}

The $\frac{\partial h k \frac{\partial \varsigma}{\partial x}}{\partial x}$ and $\frac{\partial h k \frac{\partial \varsigma}{\partial y}}{\partial y}$ terms form the convective storage terms in the continuity equation.

$k \frac{\partial \varsigma}{\partial x}$ and $k \frac{\partial \varsigma}{\partial y}$ are computed as an elemental average for stability.

The weak form of these terms is integrated using integration by parts as follows:

$$
\begin{aligned}
& \int_{\Omega} \frac{\partial}{\partial x}\left(h k \frac{\partial \varsigma}{\partial x}\right) \phi_{i} d \Omega=-\int_{\Omega}\left(h k \frac{\partial \varsigma}{\partial x}\right) \frac{\partial \phi_{i}}{\partial x} d \Omega+\int_{\Gamma_{e}}\left(h k \frac{\partial \varsigma}{\partial x}\right) \phi_{i} \bullet n_{x} d \Gamma_{e} \\
& \int_{\Omega} \frac{\partial}{\partial y}\left(h k \frac{\partial \varsigma}{\partial y}\right) \phi_{i} d \Omega=-\int_{\Omega}\left(h k \frac{\partial \varsigma}{\partial y}\right) \frac{\partial \phi_{i}}{\partial y} d \Omega+\int_{\Gamma_{e}}\left(h k \frac{\partial \varsigma}{\partial y}\right) \phi_{i} \bullet n_{y} d \Gamma_{e}
\end{aligned}
$$

The second term on the right-hand side in Equations 23 and 24 is the line integral over the element boundary multiplied by the shape function. These terms will be discussed later in this report. The following are the final forms of the convection terms:

$x$-direction terms

$$
\begin{gathered}
-\int_{\Omega}\left(h k \frac{\partial \varsigma}{\partial x}\right) \frac{\partial \phi_{i}}{\partial x} d \Omega=-k \frac{\partial \varsigma}{\partial x} \frac{\partial \phi_{i}}{\partial x} \int_{\Omega}(h) d \Omega=-k \frac{\partial \varsigma}{\partial x} \frac{\partial \phi_{i}}{\partial x} \int_{\xi=0}^{1} \int_{\eta=0}^{1-\xi}(h)(2 \Lambda) d \eta d \xi \\
-\int_{\Omega}\left(h k \frac{\partial \varsigma}{\partial x}\right) \frac{\partial \phi_{i}}{\partial x} d \Omega=-k \frac{\partial \varsigma}{\partial x} \frac{\partial \phi_{i}}{\partial x} \int_{\xi=0}^{1} \int_{\eta=0}^{1-\xi}(h)(2 \Lambda) d \eta d \xi
\end{gathered}
$$

$y$-direction terms

$$
-\int_{\Omega}\left(h k \frac{\partial \varsigma}{\partial y}\right) \frac{\partial \phi_{i}}{\partial y} d \Omega=-k \frac{\partial \varsigma}{\partial y} \frac{\partial \phi_{i}}{\partial y} \int_{\Omega}(h) d \Omega=-k \frac{\partial \varsigma}{\partial y} \frac{\partial \phi_{i}}{\partial y} \int_{\xi=0}^{1} \int_{\eta=0}^{1-\xi}(h)(2 \Lambda) d \eta d \xi
$$




$$
-\int_{\Omega}\left(h k \frac{\partial \varsigma}{\partial y}\right) \frac{\partial \phi_{i}}{\partial y} d \Omega=-k \frac{\partial \varsigma}{\partial y} \frac{\partial \phi_{i}}{\partial y} \int_{\xi=0}^{1} \int_{\eta=0}^{1-\xi}(h)(2 \Lambda) d \eta d \xi
$$

\section{Boundary Line Integration Terms}

\section{Continuity Convective Storage/Change Terms}

The terms $\int_{\Gamma_{e}}\left(h k \frac{\partial \varsigma}{\partial x}\right) \phi_{i} \bullet n_{x} d \Gamma_{e}$ and $\int_{\Gamma_{e}}\left(h k \frac{\partial \varsigma}{\partial y}\right) \phi_{i} \bullet n_{y} d \Gamma_{e}$ are the boundary line integration terms from Equations 23 and 24, respectively. These terms are integrated based on the basis function (Figure 3) defined below:

$$
\begin{aligned}
& \phi_{1}=1-\xi \\
& \phi_{2}=\xi
\end{aligned}
$$

The integration of these terms involves one-dimensional (1D) elements as shown in Figure 3.

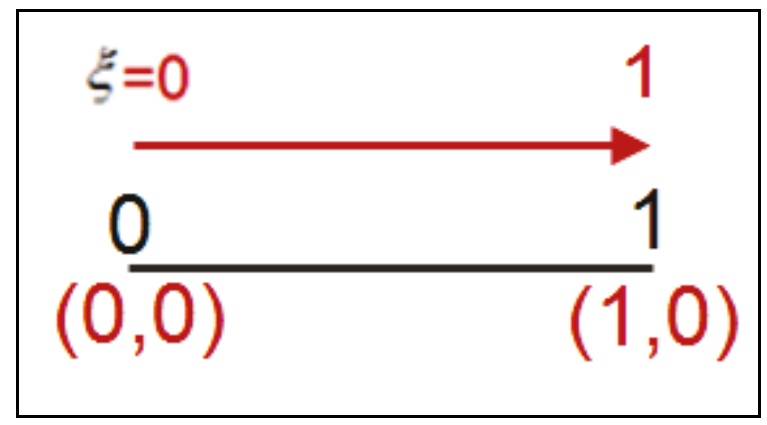

Figure 3. 1D line integral basis functions.

The basis function definitions in Equation 29 are used to expand the variables as

$$
h=\sum_{1}^{2} h_{i} \phi_{i}(x, y)=h_{1} \phi_{1}(x, y)+h_{2} \phi_{2}(x, y)
$$

which then gives the following:

$$
\begin{gathered}
\int_{\Gamma_{e}}\left(h k \frac{\partial \varsigma}{\partial x}\right) \phi_{i} \bullet n_{x} d \Gamma_{e}=k \frac{\partial \varsigma}{\partial x} n_{x} \int_{\xi=0}^{1} \phi_{i}(h) L d \xi \\
=k \frac{\partial \varsigma}{\partial x} \Delta y \int_{\xi=0}^{1} \phi_{i}(h) L d \xi
\end{gathered}
$$

where $n_{x}$ is the normal in the $x$-direction for the line segment and $\Delta y$ is $y$-ordinate distance between the nodes on the line segment. 


$$
\begin{gathered}
\int_{\Gamma_{e}}\left(h k \frac{\partial \varsigma}{\partial y}\right) \phi_{i} \bullet n_{y} d \Gamma_{e}=k \frac{\partial \varsigma}{\partial y} n_{y} \int_{\xi=0}^{1} \phi_{i}(h) L d \xi \\
=-k \frac{\partial \varsigma}{\partial y} \Delta x \int_{\xi=0}^{1} \phi_{i}(h) L d \xi
\end{gathered}
$$

where $n_{y}$ is the normal in the $y$-direction for the line segment, $L$ is the length of the line segment, and $\Delta x$ is $x$-ordinate distance between the nodes on the line segment.

BOUNDARY FILE IMPLEMENTATION: The DW equation set is activated within AdH by specification of the "OP DIF" card in the boundary conditions $\left({ }^{*} . \mathrm{bc}\right)$ file.

The card description for "OP DIF" is as follows:

\section{DW Definitions Control Cards}

\section{Diffusive Wave}

DIFFUSIVE WAVE PARAMETERS

$\begin{array}{llll}\text { Field } & \text { Type } & \text { Value } & \text { Description } \\ 1 & \text { char } & \text { OP } & \text { Card type } \\ 2 & \text { char } & \text { DIF } & \text { Physics type }\end{array}$

APPLICATION: The implementation of DW in AdH was tested on four problems: (1) a square domain with a series of depressions, (2) simulation of a flood wave, (3) a real dam break in Malpasset, France, and (4) a hypothetical dam break in North Korea.

1. Square Domain. This test specification was provided by the British Environment Agency (Neelz and Pender 2010). The test case (Test 2 in the British Environment Agency report) suggests comparing data at 16 points in the domain (locations shown in Figure 4). The modeled domain consists of numerous depressions within a perfect 2000 meter $(\mathrm{m}) \times 2000$ $\mathrm{m}$ rectangle (Figure 5). Each depression is identical in shape and $0.5 \mathrm{~m}$ in depth. Along the north to south direction a slope of 1:1500 exists, and from west to east a slope of 1:3000 exists with an approximate $2 \mathrm{~m}$ drop in elevation on the northwest to southeast diagonal.

The Computational Model Builder (CMB), a product of the U.S. Army Engineer Research and Development Center, Information Technology Laboratory, was used to create the 2D mesh of the domain. The mesh was created to have 20 m nodal spacing throughout the model domain for a total of 10,200 nodes and 20,000 elements. 


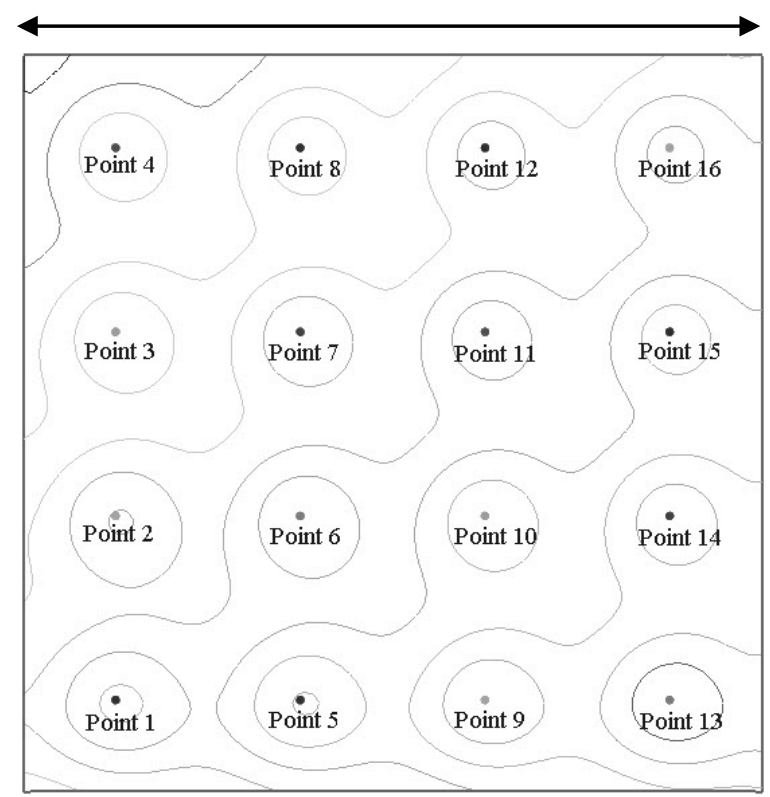

Figure 4. Plan view of the modeled domain extending from $X=0$ to $X=2000 \mathrm{~m}$ and $Y=0$ to $Y=2000 \mathrm{~m}$.

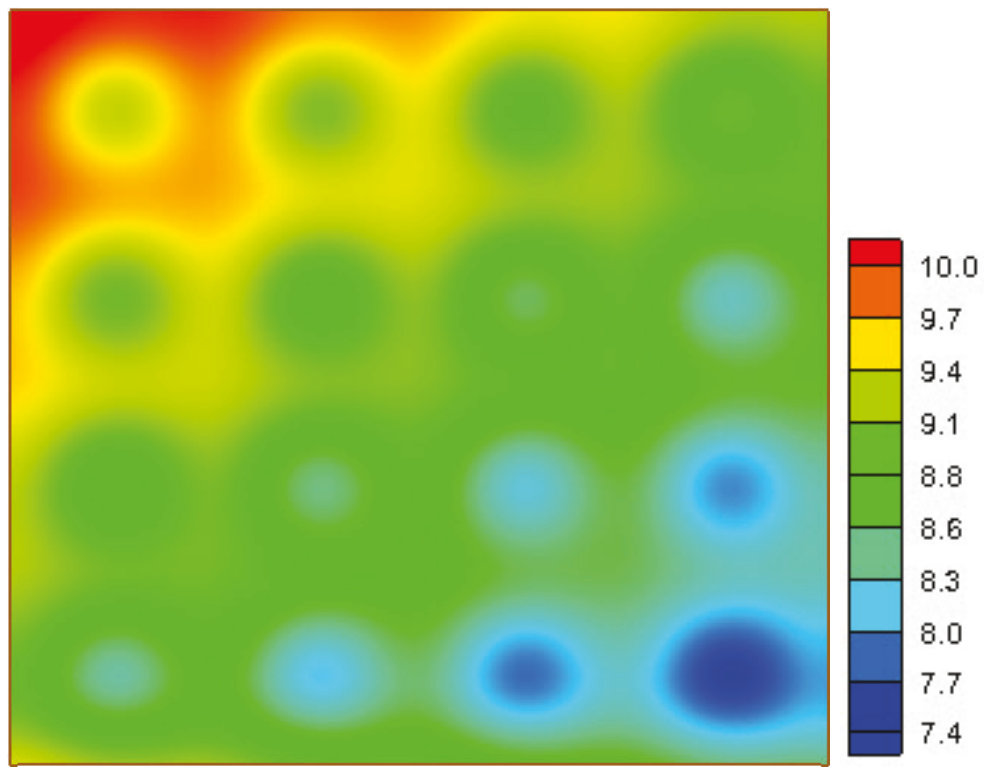

Figure 5. Contoured elevations.

A $100 \mathrm{~m}$ inflow boundary condition is specified at the upper left of the domain running south from the northwestern corner (Figure 6). The inflow hydrograph is shown in Figure 7. This inflow lasts for only the first 1.5 hours (90 minutes) while the model simulation time is for 48 hours (2880 minutes). 


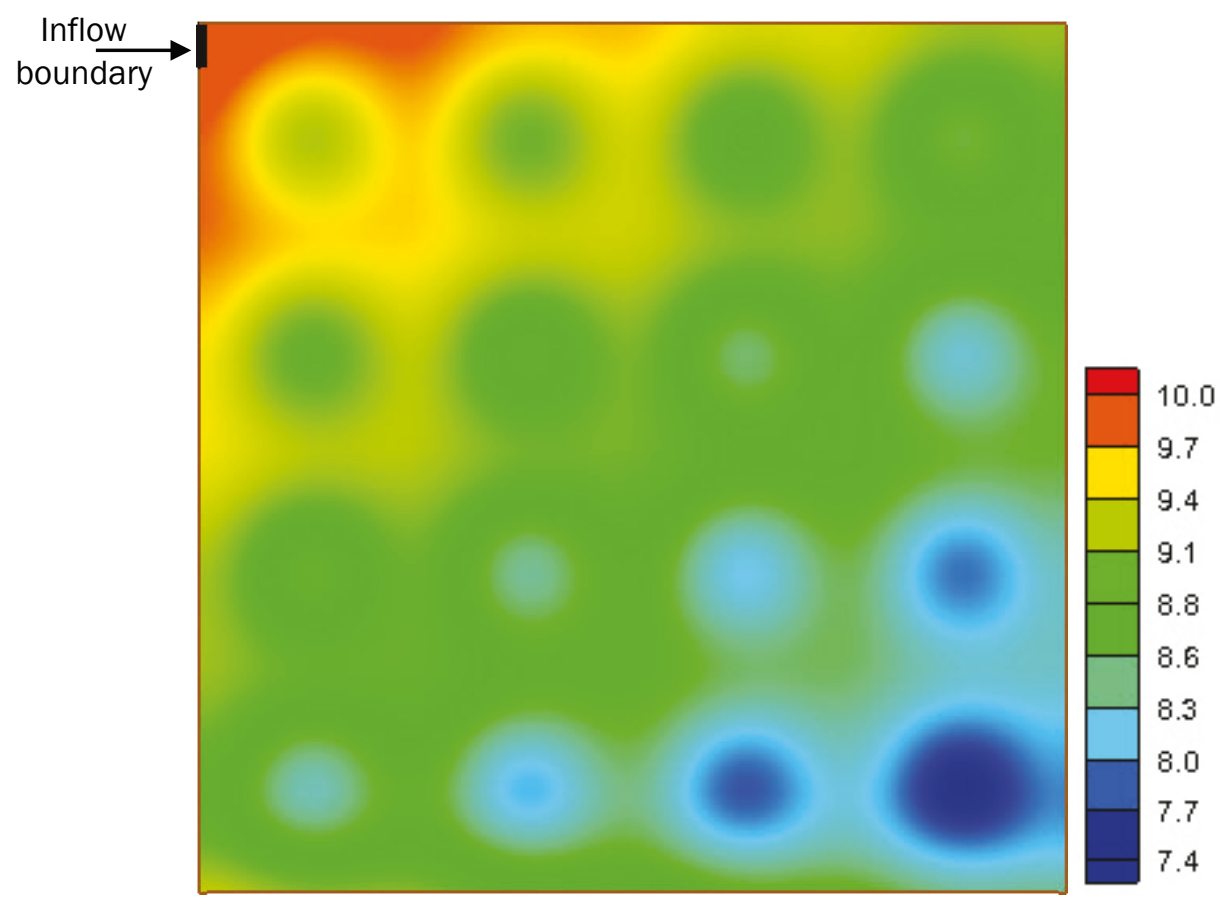

Figure 6. Inflow boundary to modeled domain.

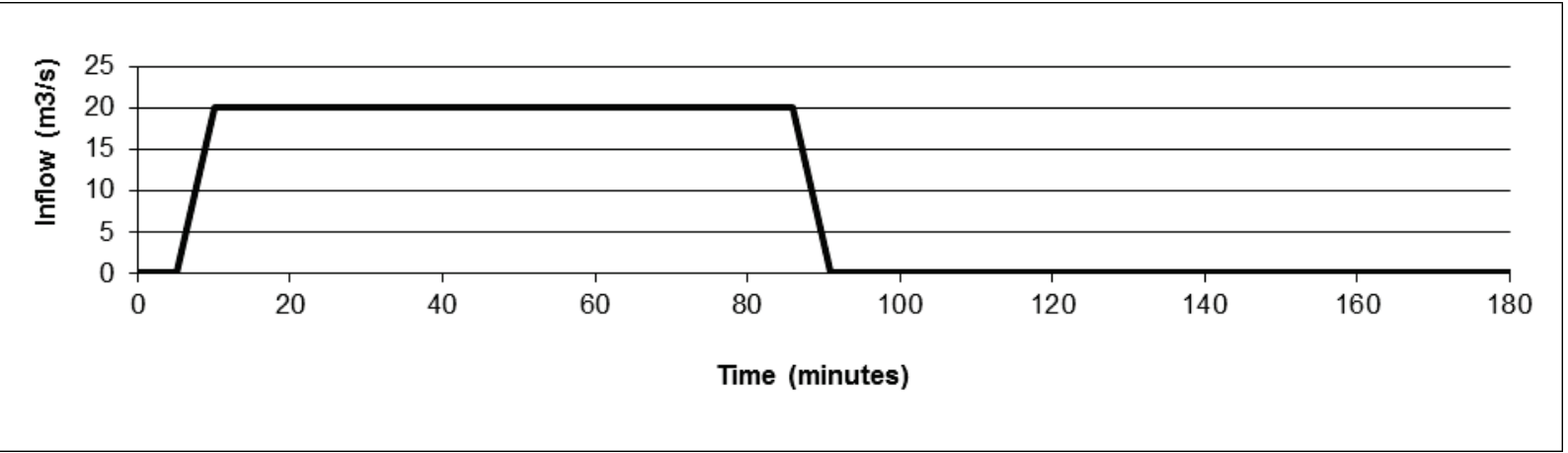

Figure 7. Flood hydrograph used as boundary condition.

A Manning's $n$ value of 0.030 was specified throughout the domain, and the simulation was executed using a constant time-step of 10 seconds (sec).

Data were extracted from the model at the Environment Agency-specified points at the center of each depression (16 points shown in Figure 4) with an output of every 300 seconds. For brevity, model and observed results are presented at locations 4 and 6, (Figures 8 and 9), and suffice to say that other locations show a similar behavior. 


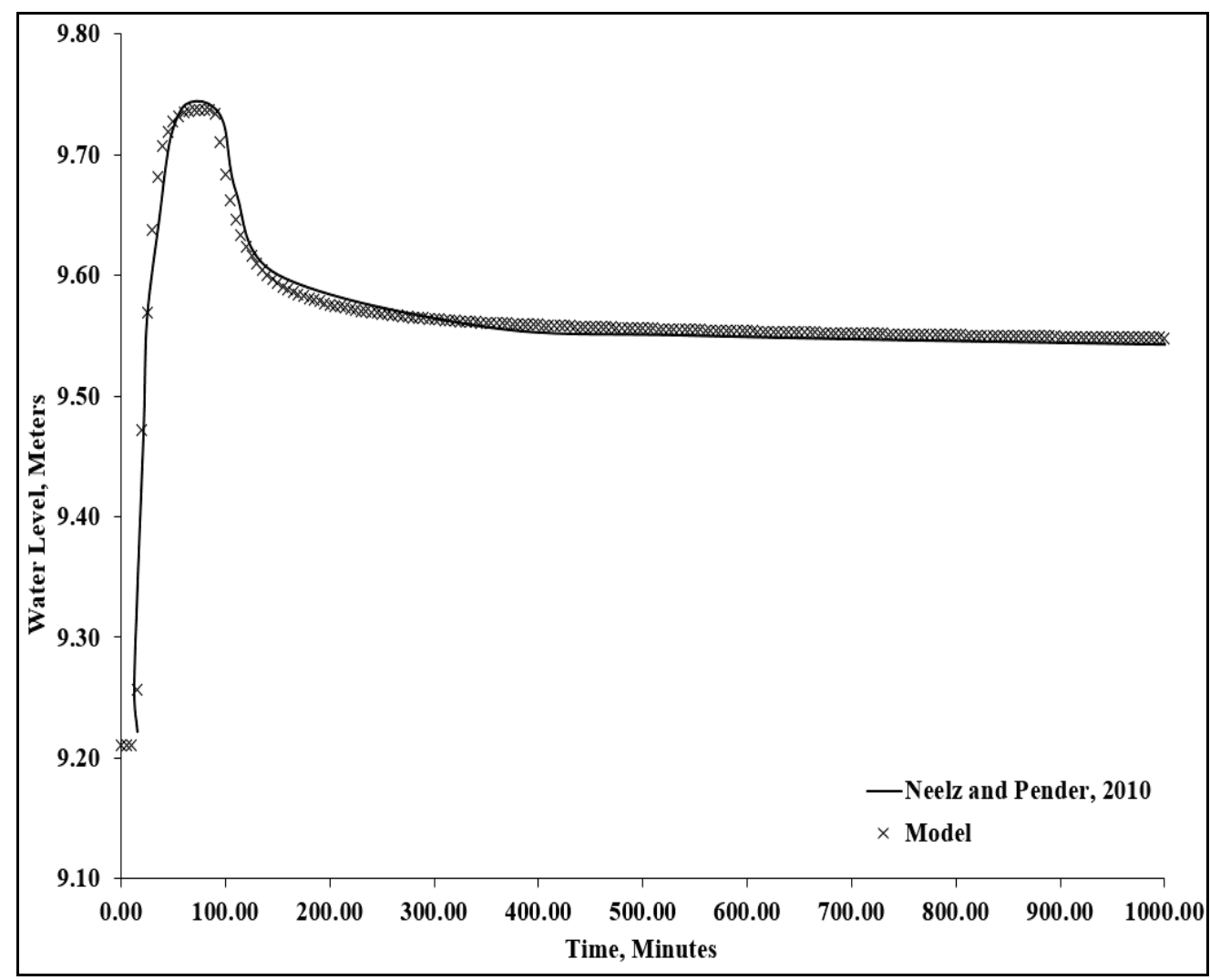

Figure 8. Water level behavior at Location 4.

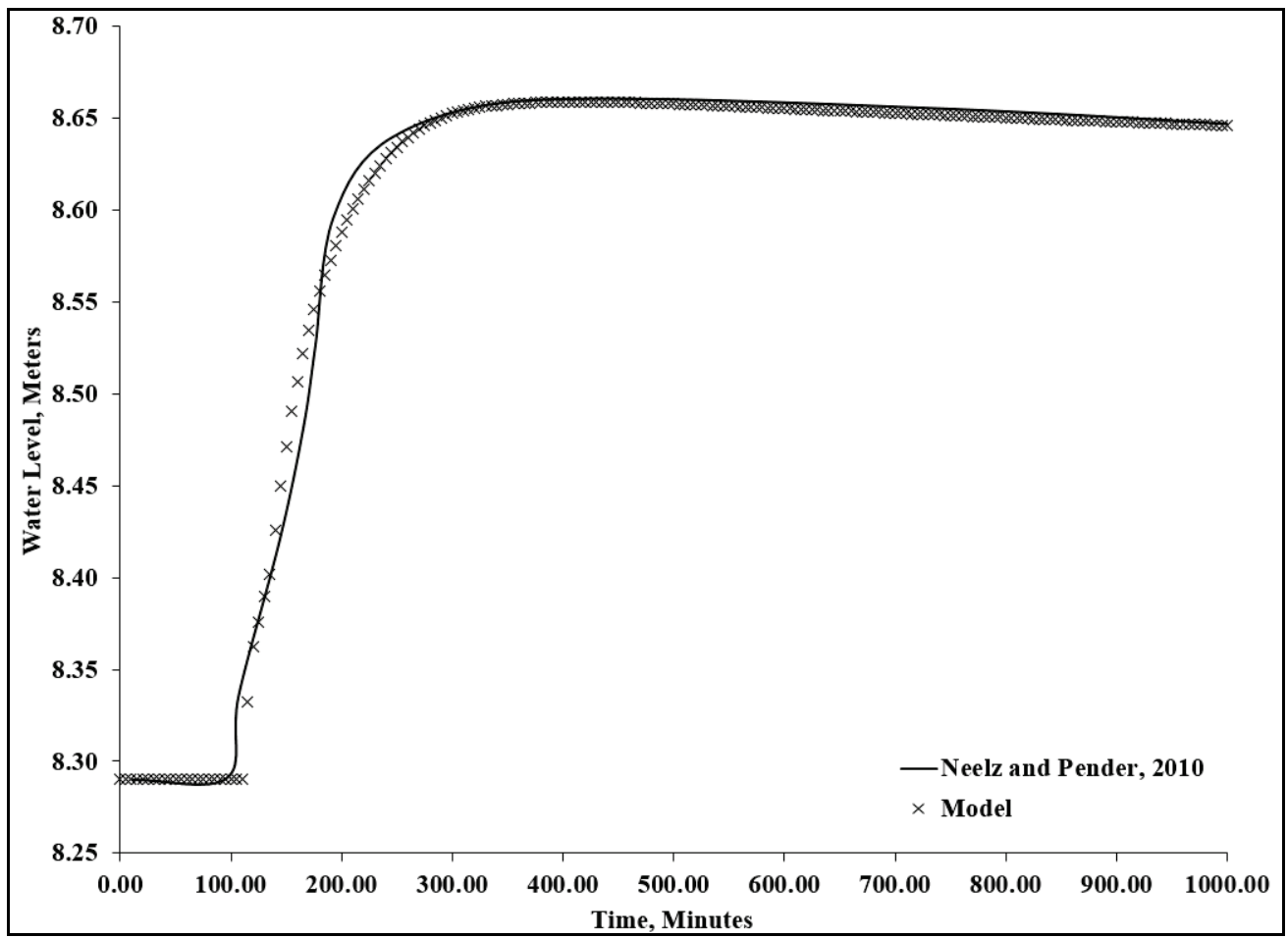

Figure 9. Water level behavior at Location 6. 
2. Flood Propagation over an Extended Floodplain. This test is based on a test (test 4) presented in Neelz and Pender (2010) and simulates the flooding caused by the failure of an embankment or overtopping. The domain consists of a $1000 \mathrm{~m} \times 2000 \mathrm{~m}$ floodplain, with inflow spread over a $20 \mathrm{~m}$ line on the left boundary of the floodplain (Figure 10).

The peak inflow is $20 \mathrm{~m}^{3} / \mathrm{sec}$ and has a base time of 300 minutes (Figure 11). The purpose of this test was to assess the ability of AdH-DW in predicting the inundation depth and the time of arrival from a flood wave.

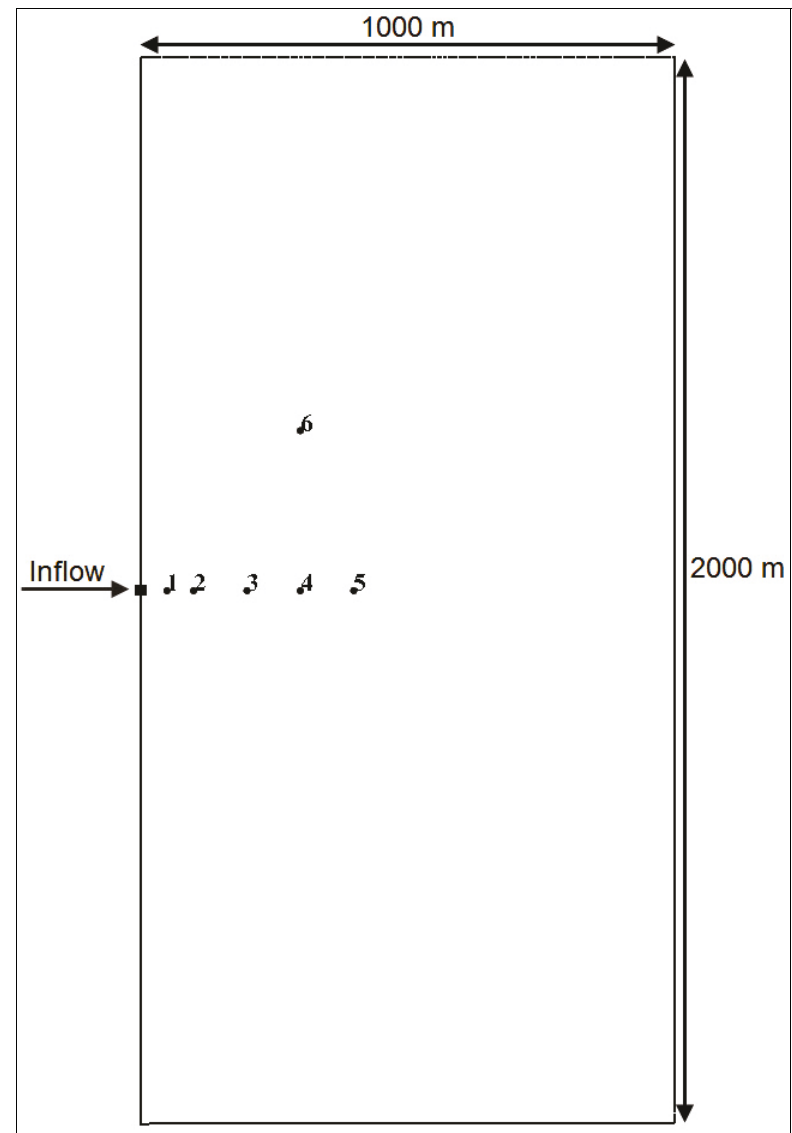

Figure 10. Test Domain and observation points.

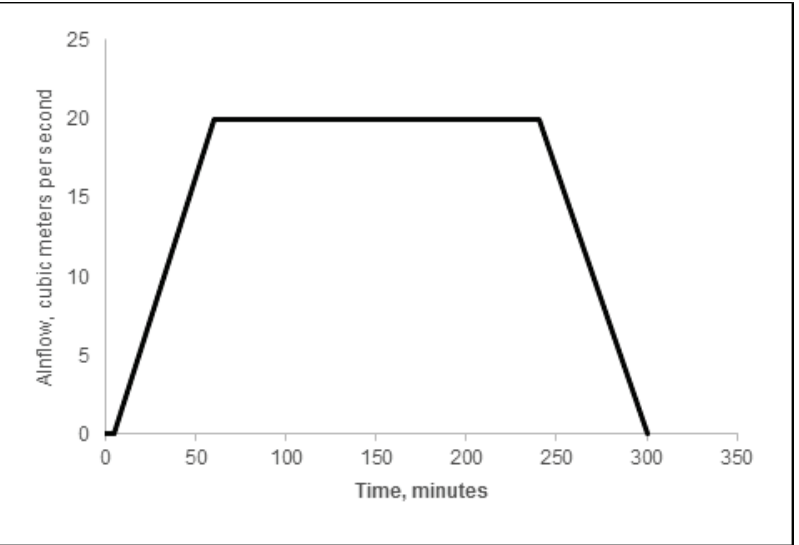

Figure 11. Inflow.

The simulation results from AdH-DW were compared at locations shown in Figure 10 to the results provided in Neelz and Pender (2010). Figures 12-17 provide a graphical comparison of these results. 


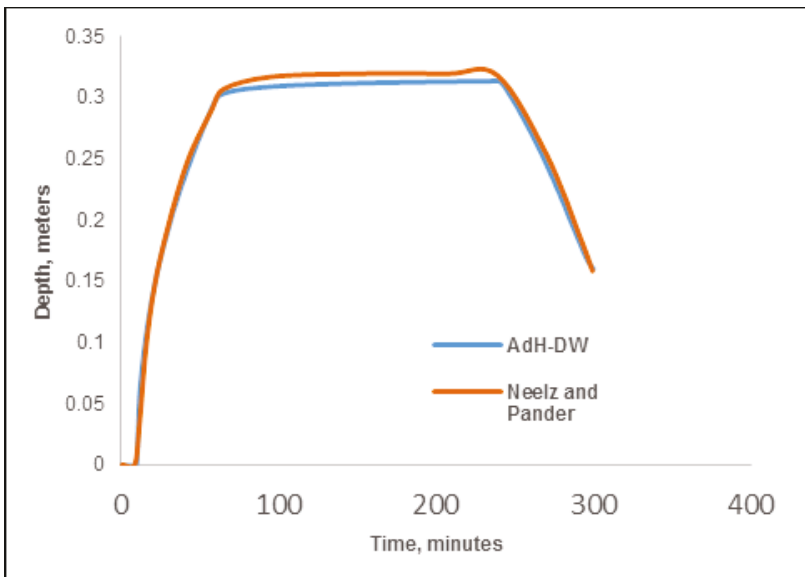

Figure 12. Water depth versus time at Point 1.

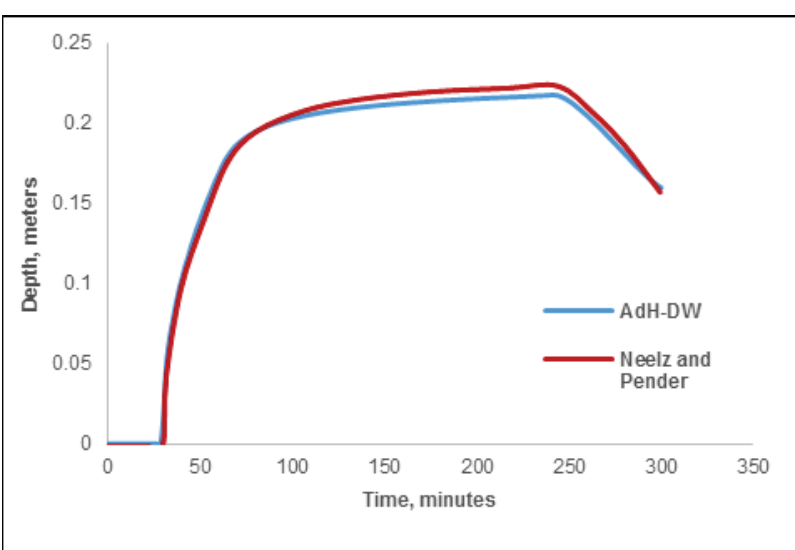

Figure 14. Water depth versus time at Point 3.

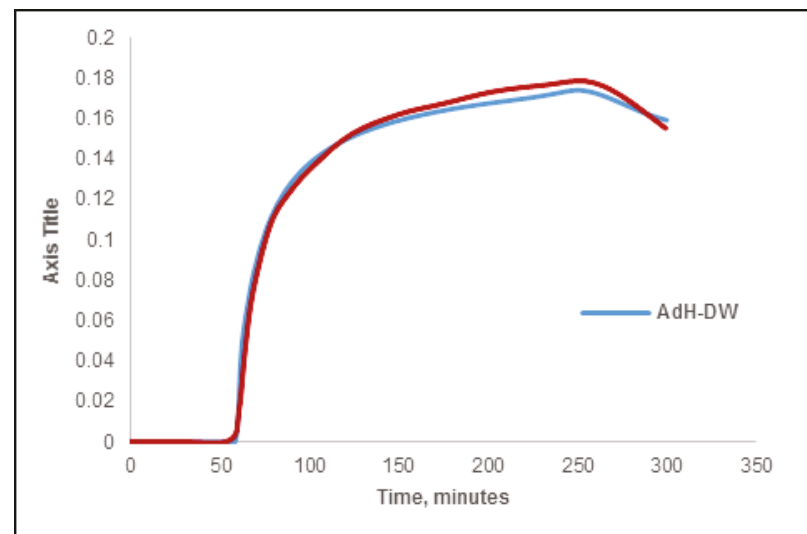

Figure 16. Water depth versus time at Point 5.

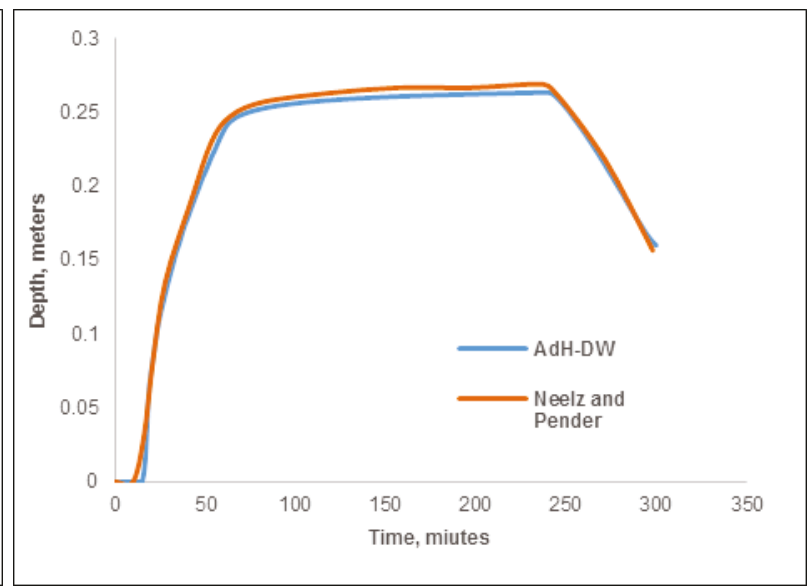

Figure 13. Water depth versus time at Point 2.

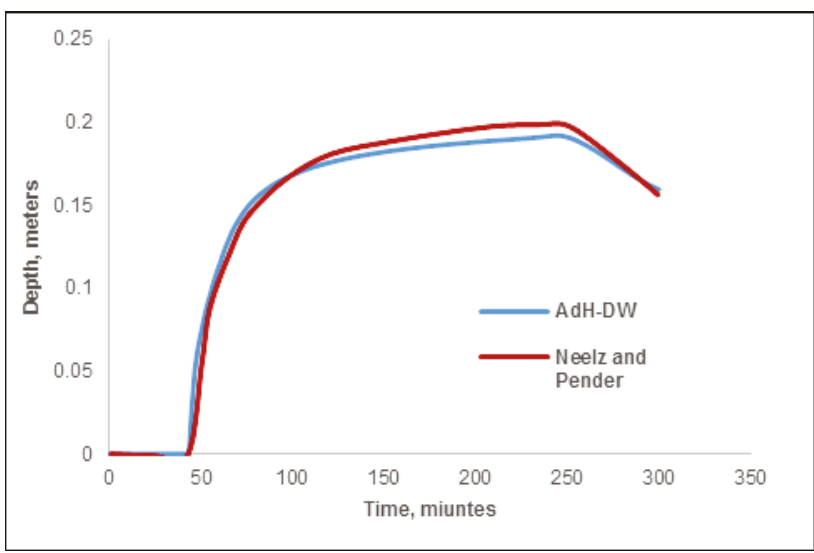

Figure 15. Water depth versus time at Point 4.

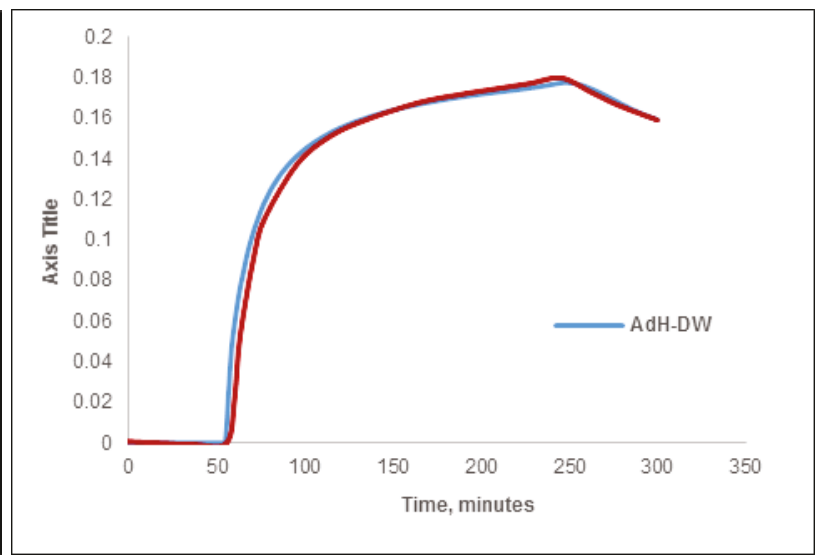

Figure 17. Water depth versus time at Point 6.

The AdH-DW simulation results compare favorably to those reported in Neelz and Pander (2010). 
3. Dam Break in Malpasset, France. The Malpasset dam failed explosively on 2 December 1959. The resulting flood wave was approximately $40 \mathrm{~m}$ high. The high water levels from the resulting flood wave were widely documented at locations provided in Figure 18. Three transformers, labeled A, B, and C, were damaged from the flood, and their loss provided arrival time information. Table 1 provides a comparison of the high water levels between the observed and model simulated values, and Table 2 provides a comparison of the flood wave arrival times.

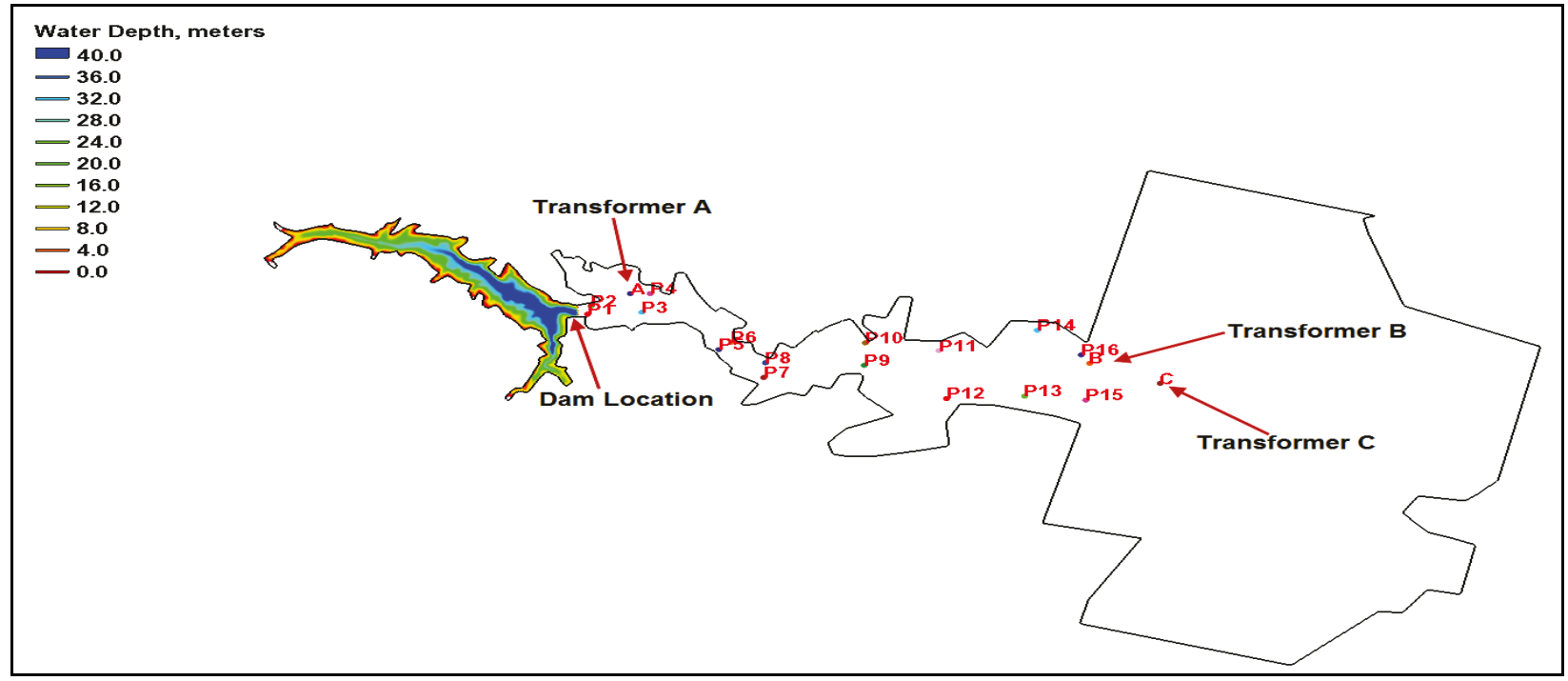

Figure 18. Malpasset Reservoir and observation locations.

Table 1. Water surface elevation comparisons.

\begin{tabular}{|l|l|l|l|l|l|l|l|l|l||}
\hline \hline \multicolumn{10}{|c|}{ Maximum Water Level (m) } \\
\hline Location & P1 & P2 & P3 & P4 & P5 & P6 & P7 & P8 & P9 \\
\hline Observed & 79.1 & 87.2 & 54.9 & 64.7 & 51.1 & 43.7 & 44.3 & 38.6 & 31.9 \\
\hline Simulated & 79.9 & 83.4 & 55.2 & 61.9 & 52.6 & 44.1 & 45.7 & 37.1 & 31.8 \\
\hline Location & P10 & P11 & P12 & P13 & P14 & P15 & P16 & & \\
\hline Observed & 40.7 & 24.1 & 24.9 & 17.2 & 20.7 & 18.6 & 17.2 & & \\
\hline Simulated & 39.5 & 24.3 & 24.7 & 16.9 & 20.6 & 18.4 & 16.4 & & \\
\hline \hline
\end{tabular}

\begin{tabular}{|l|l|l||}
\hline \multicolumn{2}{|c||}{ Table 2. Arrival time comparison. } \\
\hline \hline \multicolumn{2}{|c||}{ Travel Time (s) } \\
\hline \hline Transformer & A to B & B to C \\
\hline Observed & 1150 & 165 \\
\hline Simulated & 500 & 100 \\
\hline
\end{tabular}


AdH-DW-simulated values compare favorably with the observed water surface elevations. Since the slope of the water level and the pressure forces at the breach site are high, which violates the DW equation assumptions, the flood wave arrival times are inaccurate compared to those observed in the field.

4. Hypothetical Breach of a Reservoir in Asia. This application is an efficiency/turnaround test of the AdH-DW to simulate a large-scale dam break. The simulation development utilized the tool suite available within CMB. The "grab cut" tool available within CMB was used to rapidly create the land-water delineation for the simulation, and the "filigree" meshing option was used for the generation of AdH-DW mesh. Figure 19 shows the general domain extents (visualization was performed within the SMS software suite).

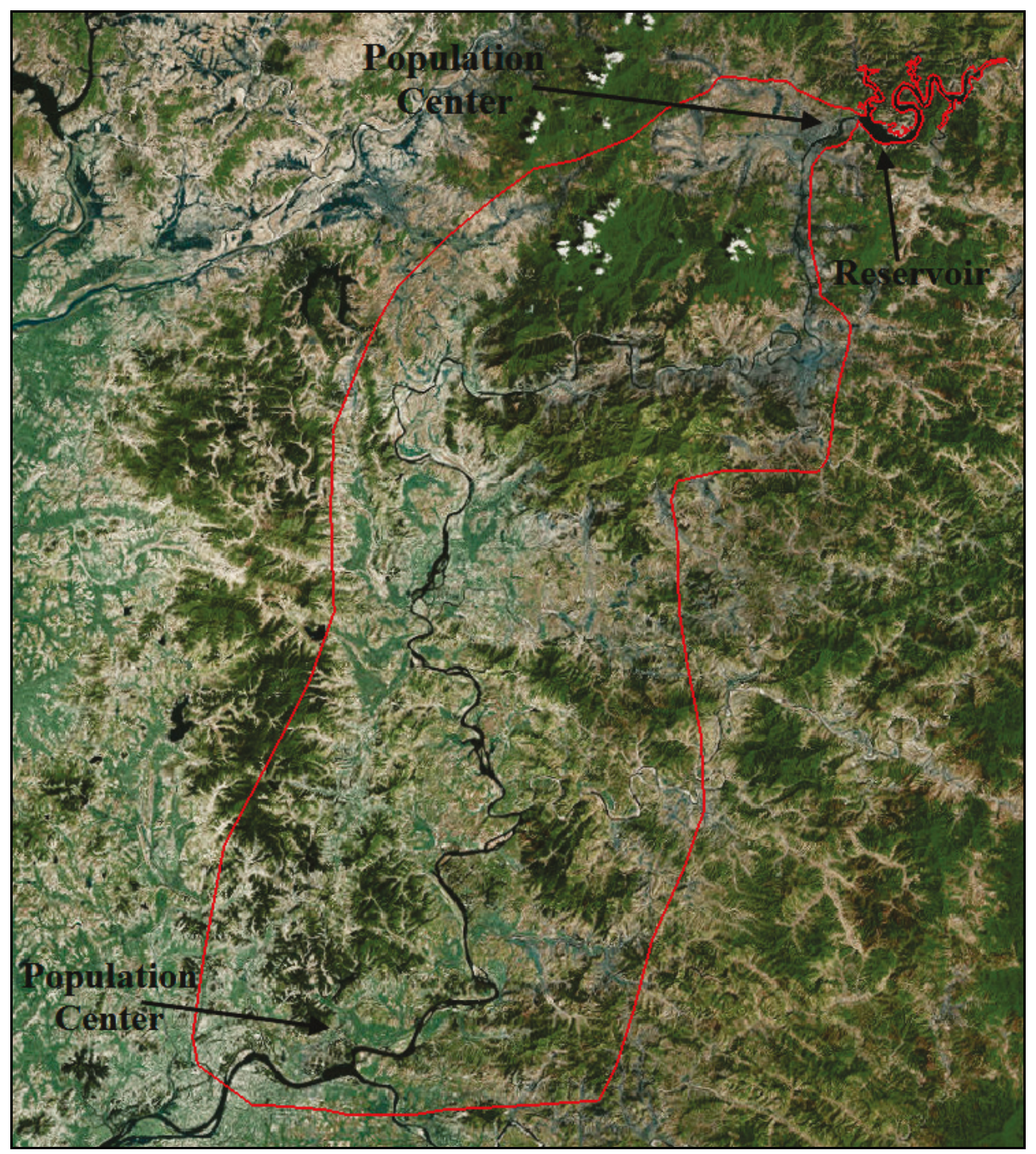

Figure 19. Model domain (red indicates mesh boundary). 
Shuttle Radar Topography Mission data obtained using the Environmental Simulator program's QUEST data services library were used as the underlying elevation data for the simulation (Figure 20).

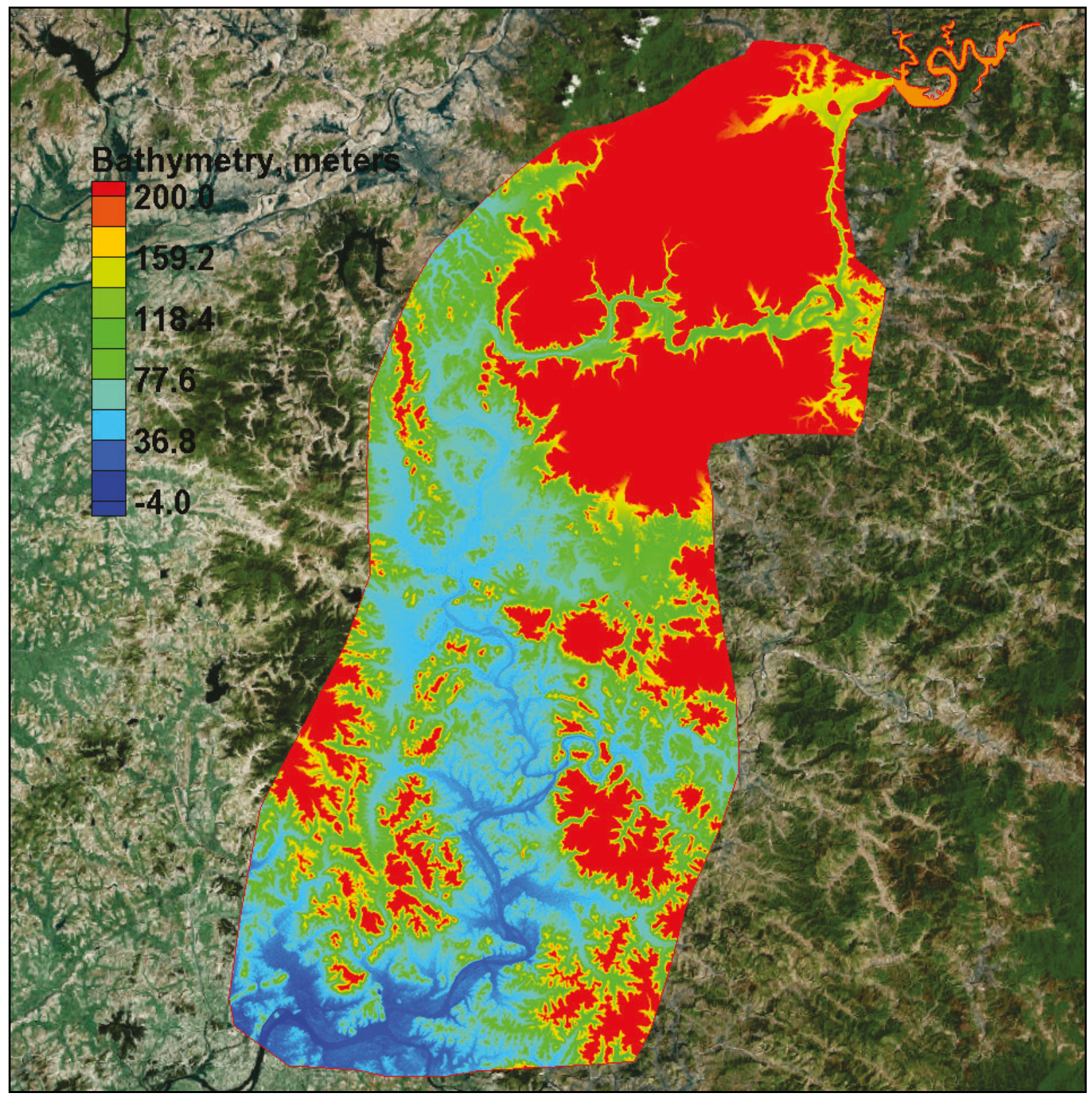

Figure 20. Underlying model bathymetry.

The exact reservoir capacity for the reservoir is unknown, and therefore AdH-DW simulations were performed with constant reservoir depths of $5 \mathrm{~m}$ and $10 \mathrm{~m}$ (Figure 21). 


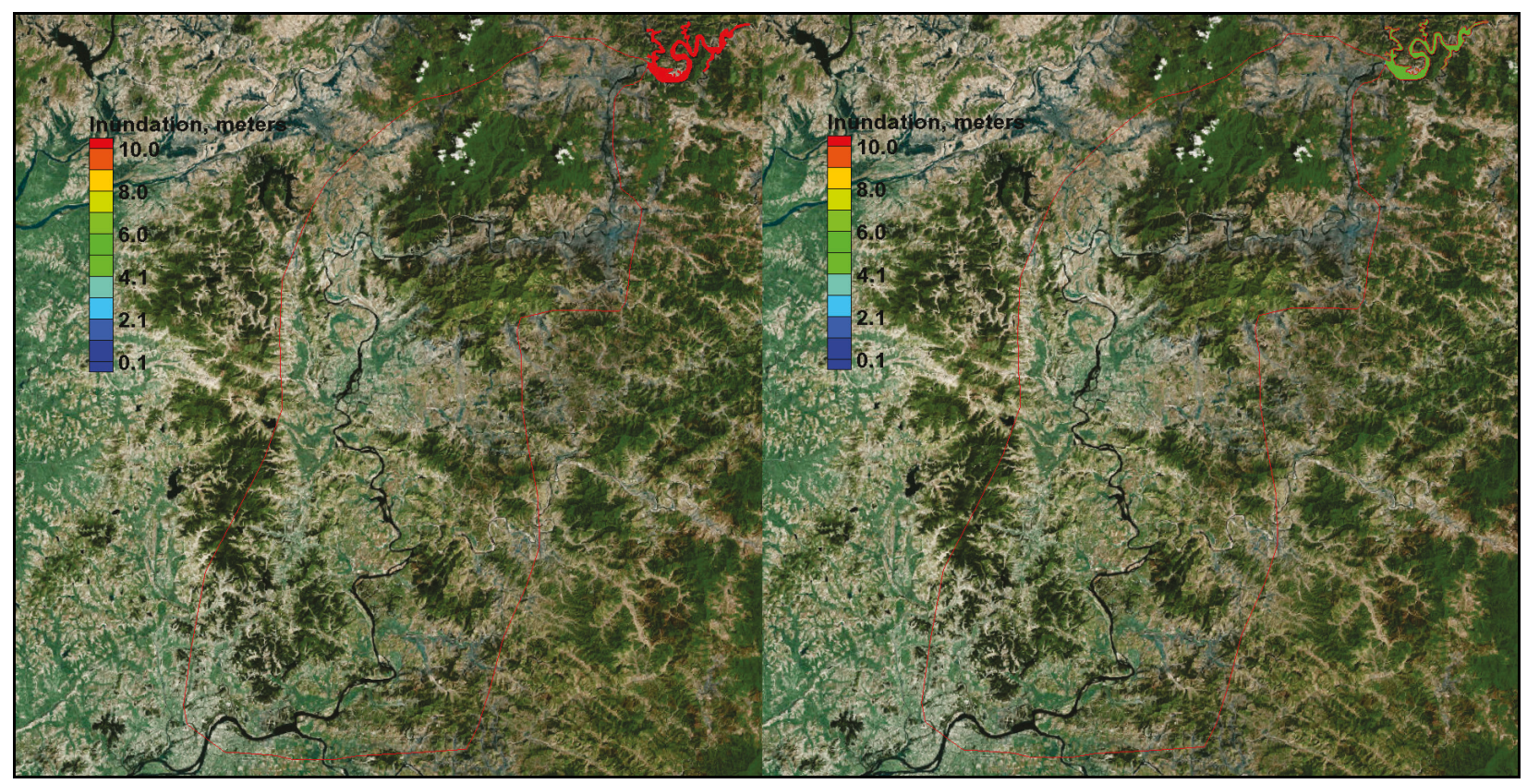

Figure 21. Model initial reservoir depth (left panel: $10 \mathrm{~m}$ initial depth; right panel: $5 \mathrm{~m}$ initial depth).

Figures 22 and 23 present the inundation at days 1 and 3 after the breach.

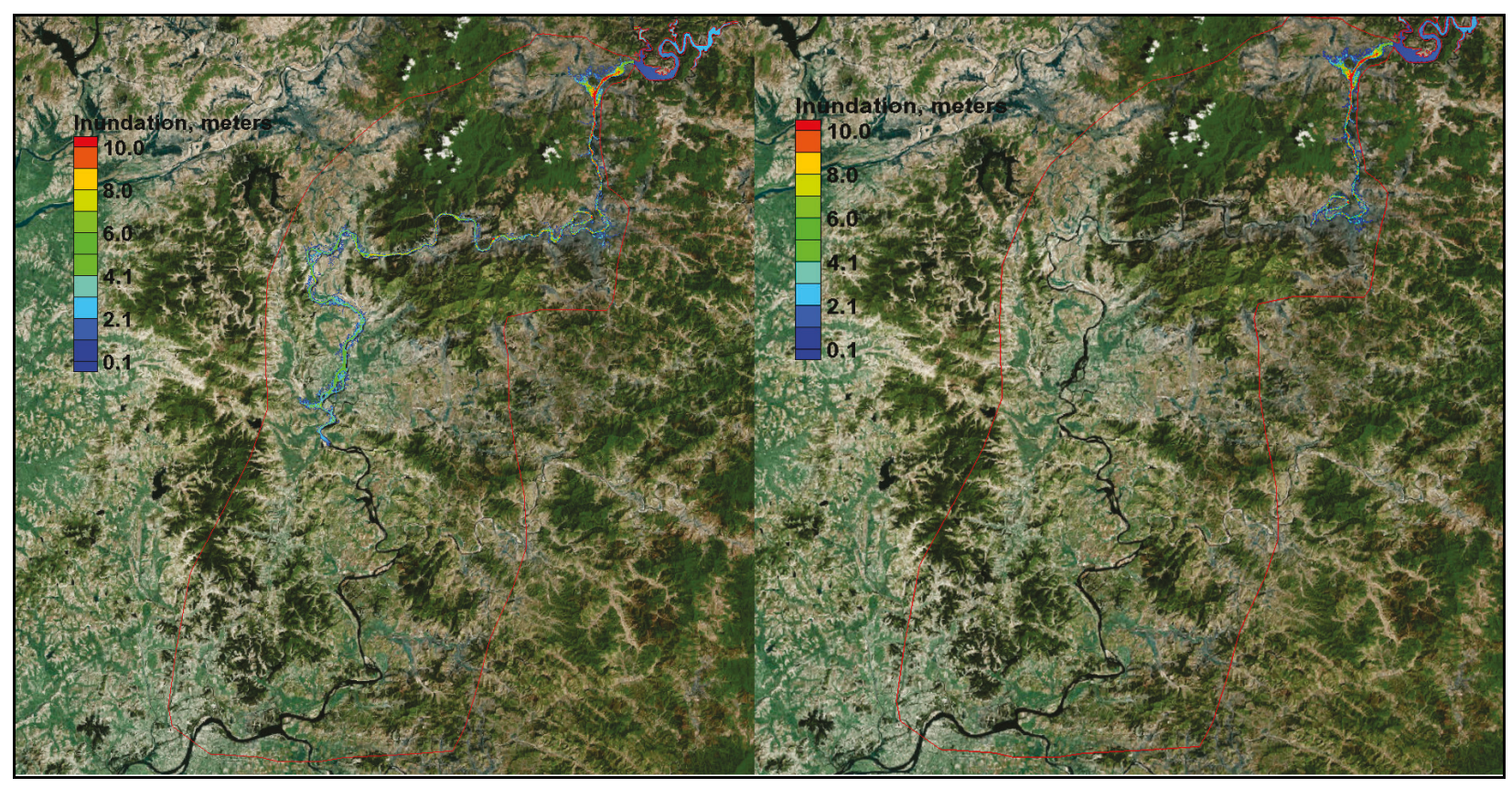

Figure 22. Model inundation 1 day after breach (left panel: $10 \mathrm{~m}$ initial depth; right panel: $5 \mathrm{~m}$ initial depth). 


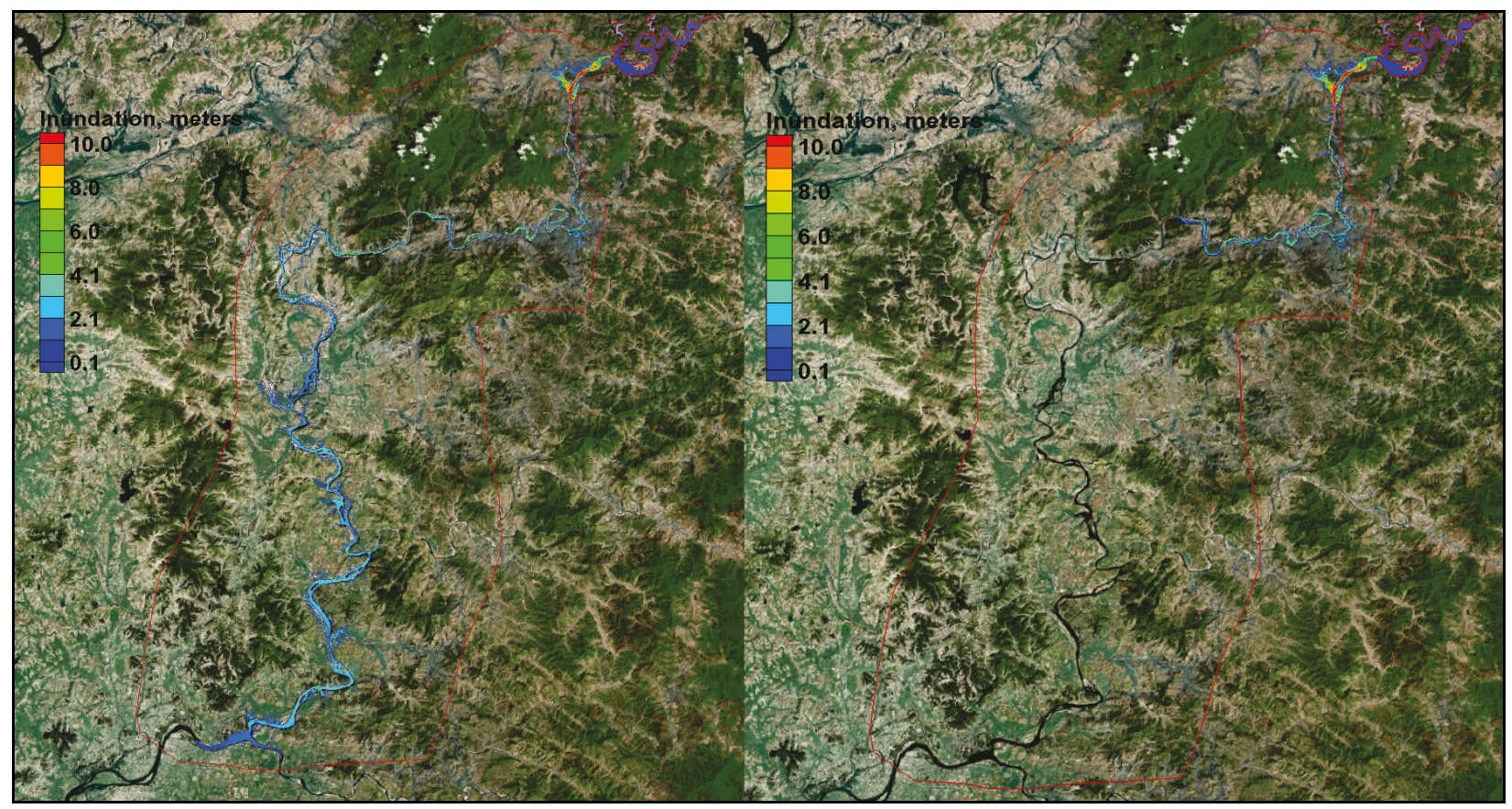

Figure 23. Model inundation 3 days after breach (left panel: $10 \mathrm{~m}$ initial depth; right panel: $5 \mathrm{~m}$ initial depth).

SUMMARY AND CONCLUSIONS: This CHETN describes the development, and testing of the diffusive wave module for the AdH hydrodynamic code. The implementation was tested on three cases: (1) a test problem consisting of a series of depressions, (2) a test involving the flooding of a floodplain, (3) Malpasset Dam-break problem, and (4) hypothetical dam break in Asia. The AdH-DW accurately replicated the inundation extents for Test 1, Test 2, and the Malpasset, France, application and was both stable, efficient, and quantitavely accurate for a hypothetical dam break application in Asia. As expected, the flood wave arrival time was inaccurate for the Malpasset dam break test given that the DW equations are inaccurate at the breach site due to the assumptions of the DW equations.

POINTS OF CONTACT: This CHETN was prepared as part of the Environmental Simulator (ES) work package of the Engineered Resilient Systems (ERS) program of the U.S. Army Corps of Engineers. The POC for technical inquiries is Dr. Gaurav Savant (gaurav.savant@erdc.dren.mil). For information about the ES work package, please contact the ES work package manager, Dr. Jeffrey Hensley (Ueffrey.L.Hensley@erdc.dren.mil).This technical note should be referenced as follows:

Savant, G., C. J. Trahan, and T. O. McAlpin. 2018. Rapid dam break and flooding simulations using the Diffusive Wave (DW) Module of Adaptive Hydraulics (AdH). ERDC/CHL CHETN-IV-112. Vicksburg, MS: U.S. Army Engineer Research and Development Center. $\underline{\text { ttp: }: / / d x . d o i . o r g / 10.21079 / 11681 / 29351 . ~}$ 


\section{REFERENCES}

Neelz, S., and G. Pender. 2010. Benchmarking of 2D hydraulic modeling packages. Great Britain: Environment Agency.

Prestininzi, P. 2008. Suitability of the diffusive model for dam break simulation: Application to a CADAM experiment. Journal of Hydrology 361(1-2): 172-185.

Savant, G., C. Berger, T. O. McAlpin, and J. N. Tate. 2011. Efficient implicit finite-element hydrodynamic model for dam and levee breach. Journal of Hydraulic Engineering 137(9): 1105-1018.

NOTE: The contents of this technical note are not to be used for advertising, publication, or promotional purposes. Citation of trade names does not constitute an official endorsement or approval of the use of such products. 